\title{
Association of Apolipoprotein E Polymorphism With Risk of Metabolic Syndrome: A Case-Control Study And Meta- Analysis
}

\section{Mahdi Safarpour}

Shahid Beheshti University of Medical Sciences: Shaheed Beheshti University of Medical Sciences

\section{Bahareh Sedaghati-khayat}

Shahid Beheshti University of Medical Sciences: Shaheed Beheshti University of Medical Sciences

\section{Leila Najd Hassan Bonab}

Shahid Beheshti University of Medical Sciences: Shaheed Beheshti University of Medical Sciences

Mohammad-Sadegh Fallah

Shahid Beheshti University of Medical Sciences: Shaheed Beheshti University of Medical Sciences

Ahmad Ebrahimi

Shahid Beheshti University of Medical Sciences: Shaheed Beheshti University of Medical Sciences

\section{Fereidoun Azizi}

Shahid Beheshti University of Medical Sciences: Shaheed Beheshti University of Medical Sciences

\section{Maryam S Daneshpour ( $\nabla$ daneshpour@sbmu.ac.ir)}

Shahid Beheshti University of Medical Sciences

\section{Research}

Keywords: apolipoprotein E, metabolic syndrome, TCGS, meta-analysis

Posted Date: August 31st, 2021

DOI: https://doi.org/10.21203/rs.3.rs-476952/v1

License: (c) (i) This work is licensed under a Creative Commons Attribution 4.0 International License. Read Full License 


\section{Abstract}

Background: This study aimed to assess the genetic association between Apolipoprotein E (APOE) polymorphisms and susceptibility to metabolic syndrome (MetS) among the Tehran cardiometabolic genetic study (TCGS) participants. The results were then compared with similar studies in other populations using meta-analysis.

Methods: the metabolic syndrome was defined according to the Joint Interim Statement (JIS) criteria. A total of 12282 subjects (5696 cases with metabolic syndrome and 6586 healthy controls) were genotyped using Human OmniExpress-24-v1-0 BeadChip. Subsequently, PubMed, Scopus, and Google Scholar databases were searched using relevant Medical Subject Headings (MeSH) terms to collect all studies that examined the association between polymorphisms of the APOE gene and risk of MetS.

Results: the presence of the APOE- $\varepsilon 4$ allele could not significantly increase the risk of MetS in the carriers of this allele compared to individuals lacking the risk allele in the TCGS population ( $\mathrm{OR}=1.11,95 \% \mathrm{Cl}$ : 0.92 to 1.32 ). The metaanalysis results also indicated no significant association between the APOE- $\varepsilon 4$ allele and the risk of MetS.

Conclusion: the frequency of the $\varepsilon 4$ allele was almost same in the cases and the controls. Therefore, this allele cannot be considered as biomarker to predict the MetS susceptibility in the TCGS population or other populations with different genetic pools.

\section{Introduction}

Metabolic syndrome is a polygenic multifactorial metabolic disorder resulting from a cluster of metabolic risk factors that directly increase the risk of heart disease, stroke, and diabetes. The co-occurrence of at least three out of five medical conditions could diagnose this syndrome: abdominal (central) obesity, elevated blood pressure, elevated fasting plasma glucose, high serum triglycerides, and low high-density lipoprotein (HDL).

It is shown that several genetic factors are involved in the pathogenesis of this disease that can regulate the risk factors of metabolic syndrome. Current investigations are more devoted to genes responsible for lipoprotein metabolism. Among these genes, the APOE gene is introduced as one of the candidate genes. The APOE gene, mapping to the 19q13 chromosomal region, encodes cholesterol and other lipid transporters[1]. The gene product binds to a specific liver and peripheral cell receptor and is essential for the triglyceride-rich lipoprotein constituents' normal catabolism. Two single-nucleotide polymorphisms within exon four at codons 112 and 158 produce three common isoforms of the APOE gene designated as $\varepsilon 2, \varepsilon 3, \varepsilon 4[2]$. Combining these alleles make six different genotypes. The APOE-e2 (Arg158RCys) is shown to be associated with decreased levels of total and low-density lipoprotein (LDL) cholesterol. In contrast, APOE-e4 (Cys112RArg) is concerning increased cholesterol levels [3, 4],[5]'[6]. The APOE-e3 is the common form with cysteine and arginine at residues 112 and 158, respectively, required for normal APOE functioning. Therefore, this genotype is considered as reference genotype, and persons who have this genotype are regarded as having a risk level of 1.0.

In addition to the impact on the serum lipid profile, blood pressure levels [7], development of type 2 diabetes mellitus [8], and neurodegenerative disorders [9], polymorphisms of the APOE gene also acting in the occurrence and development of metabolic syndrome[10]. Based on the epidemiologic studies, the $\varepsilon 4$ allele carriers are particularly predisposed to develop MetS. In contrast, the presence of the $\varepsilon 2$ allele is shown to play a protective role by lowering LDL concentration. However, other studies reported opposite findings[11]. This polymorphic characteristic makes the association between the $\varepsilon 2$ and $\varepsilon 4$ allele and metabolic syndrome remain vague. Due to the different and complex associations of $A P O E$ alleles and genotypes in determining MetS risk, achieving a broad conclusion is of high

Page 2/16 
importance. Therefore, this study was carried out to investigate if different $A P O E$ genotypes could determine the susceptibility to MetS.

\section{Materials And Methods}

\section{Study population}

This study was conducted in the Tehran Cardio-metabolic Genetic Study (TCGS) [16]. In brief, the TCGS is a part of an ongoing cohort study, the Tehran lipid, and glucose study (TLGS) [17], in which subjects genotyped and followed up for cardio-metabolic risk factors every three years since 1999. For the current study, the sample consists of 12282 individuals without any degree of relatedness (5409 men and 6873 women) that assigned to the case group if they were diagnosed with metabolic syndrome in two or more two phases of study according to the JIS criteria [18]. We excluded 2873 individuals for whom the genotype information for targeted APOESNPs was not available. All contributors signed informed written consent. The ethics committee of the Research Institute for Endocrine Sciences approved the study. All methods of the current study followed relevant guidelines and regulations.

\section{Clinical, anthropometric, and laboratory measurements}

Data were collected using interviews, physical examinations, and laboratory measurements. Details of measurement of MetS components, including waist circumference (WC), fasting plasma glucose (FPG), systolic and diastolic blood pressure (SBP and DBP, respectively), high-density lipoprotein cholesterol (HDL-C), and triglycerides (TG), have been described elsewhere[19]. Components of MetS were: waist circumference ( $\geq 95 \mathrm{~cm}$ for both Iranian men and women), elevated blood pressure (systolic/diastolic blood pressure $\geq 85 / 130 \mathrm{~mm} \mathrm{Hg}$ ), and low HDL- cholesterol ( $<40 \mathrm{mg} / \mathrm{dl}$ in men and $<50 \mathrm{mg} / \mathrm{dl}$ in women), elevated triglycerides (high TG) ( $\geq 150 \mathrm{mg} / \mathrm{dl}$ ), and high glucose ( $\geq 100 \mathrm{mg} / \mathrm{dl}$ ) [18]. Drug treatments, including anti-hypertensive drugs, lipid medication, and diabetes medication, were considered as separate binary variables.

APOE Genotyping: Samples were washed with lysis buffer where PBS and RBCs were separated. Then, through a salting-out method, DNA was extracted from the WBCs, and the cell extracts were stored at -20॰C [16]. Quantitative and qualitative assessments on the extracted DNA performed by electrophoresis and spectrophotometry. Genomic samples assayed by Human OmniExpress-24-v1-0 (Illumina Inc., San Diego, CA) chip for genotyping marker identification [17]. The genotype information for rs7412 and rs429358 extracted from the imputed dataset for all individuals included in this study.

\section{Statistical Analysis}

Descriptive statistics used for population characteristics and data were shown as mean \pm SD for normally distributed variables and as percentages for categorical variables. The student's t-test evaluated differences between case and control groups for normally distributed data. The distribution of the triglyceride was skewed, and the comparison performed using Mann-Whitney U-test. Analysis of categorical variables performed by Chi-square and Fisher's exact tests for contingency tables. The $2 \times 2$ contingency table was used to estimate the odds of MetS in $\varepsilon 4$ carriers compared to $\varepsilon 2$ carriers. The estimated odds ratio (OR) was crude without any adjustment for possible confounders.

Allele frequency and Hardy-Weinberg equilibrium computed using Power Marker software. The genotype frequency of E2E2 and E2E4 is compared in case and control groups. Also, the genotypes are classified into two main groups, including the $\varepsilon 2$ and $\varepsilon 4$ carriers. The $\varepsilon 2$ group comprises subjects with $\varepsilon 2 / \varepsilon 2$ or $\varepsilon 2 / \varepsilon 3$ genotypes, and the $\varepsilon 4$ group was comprised of the subjects with $\varepsilon 3 / \varepsilon 4$ and $\varepsilon 4 / \varepsilon 4$ genotypes. The subjects with an $\varepsilon 2 / \varepsilon 4$ genotype were excluded 
because of the $\varepsilon 2$ and $\varepsilon 4$ alleles' potentially opposite effects. The average levels of anthropometric and laboratory parameters compared among the three genotype groups $(\varepsilon 2, \varepsilon 3$, and $\varepsilon 4$ allele carriers) by using the student's t-test

\section{Sources and searches}

A systematic search carried out using PubMed, Scopus, and Google scholar databases without any time limitation. The keywords applied for the search for genotype were: APOE, Apolipoprotein E, apolipoprotiene2, apolipoprotiene3, apolipoprotiene4, rs7412, and rs429358. For the disease, the search term was: metabolic syndrome, metabolic syndrome X, MetS, syndrome X. References from published prospective studies, relevant reviews, and previous metaanalyses were hand searched for additional studies not identified in the database search.

\section{Study selection}

Qualified studies were selected if they meet the following criteria: 1) were case-control or genome-wide association study (GWAS); 2) stratified subjects based on the presence or absence of the metabolic syndrome using the NCEP, ATP III, WHO, IDF, AACE, or JIS definitions; 3) as count data, or as odds ratio (OR) with a corresponding measure of \%95 confidence interval; 4) carried on human subjects; and 5) were published in the English language. Studies investigating more than one definition of metabolic syndrome were also eligible for inclusion. Studies not meeting these criteria were excluded (Supplementary Table 1).

\section{Data extraction}

Two groups of reviewers (two reviewers in each group) independently extracted data using standardized and modified data extraction forms in terms of any disagreements, the issue resolve by agreement or, when needed, consulted by a third reviewer [20]. According to each definition, reviewers pull out information on study design, including the number of participants with and without the metabolic syndrome. Outcomes data presented as count data, adjusted or nonadjusted risk estimates (OR) with corresponding measures of variance, or multivariable-adjusted risk estimates extracted for participants with and without the metabolic syndrome (Supplementary Table 2).

\section{Data synthesis and analysis}

The results of the included studies were synthesized using random-effects meta-analysis, and synthesized results presented as ORs with corresponding 95\% confidence intervals (Cls) (Supplementary Table 3 (1-3)). The heterogeneity assessed using $\mathrm{I}^{2}$ statistics. This meta-analysis was conducted only for studies that reported outcomes as count data or studies reported outcomes as risk estimates only (OR). Other studies not meeting the mentioned criteria were excluded from this analysis. Moreover, in the general population, the metabolic syndrome risk associated with the $A P O E$ genotypes was estimated without considering different metabolic syndrome definitions.

On the other hand, the overall metabolic syndrome risk was assessed when all definitions pooled. Funnel plots were constructed to assess the possible presence of publication bias. Besides, Begg's and Egger's tests were used to assess any shreds of evidence for publication bias. The sensitivity analysis was carried to explore the impact of excluding or including studies in a meta-analysis based on the sample size. All analyses were conducted using the STATA software version [19].

\section{Results}

The demographic and biochemical characteristics of the 12282 individuals recruited from the TCGS participants are shown in Table 1. Based on the results, age, BMI, hip, waist, waist to hip ratio, total cholesterol, serum LDL and HDL, 
systolic and diastolic blood pressure, and glucose levels were significantly different between the case and control groups. The summary of demographic and clinical characteristics of males and females presented in Table 2. BMI was significantly higher in women $(27.97 \pm 5.59)$ than in men $(26.67 \pm 4.70)$, and weight, height, waist to hip ratio, and serum HDL also showed significant differences between different gender (Table 2).

Table 1

Clinical, anthropometric, and laboratory measurements in case and control groups

\begin{tabular}{|c|c|c|c|c|}
\hline Parameters & $\begin{array}{l}\text { Total }(n= \\
12282)\end{array}$ & $\begin{array}{l}\text { With MetS }(n= \\
5696)\end{array}$ & $\begin{array}{l}\text { Without MetS }(n= \\
6586)\end{array}$ & $\begin{array}{l}\text { P- } \\
\text { value }\end{array}$ \\
\hline Age (year) & $\begin{array}{l}42.96 \pm 18.59 \\
\text { a) }\end{array}$ & $55.69 \pm 4.12$ & $36.74 \pm 17.30$ & $<0.001$ \\
\hline Body mass index $\left(\mathrm{kg} / \mathrm{m}^{2}\right)$ & $27.40 \pm 5.26$ & $30.14 \pm 4.85$ & $25.01 \pm 4.35$ & $<0.001$ \\
\hline Weight (kg) & $71.82 \pm 15.12$ & $79.31 \pm 13.96$ & $68.13 \pm 14.29$ & $<0.001$ \\
\hline Height $(\mathrm{cm})$ & $161.85 \pm 10.79$ & $161.06 \pm 10.32$ & $162.24 \pm 11.01$ & 0.304 \\
\hline Hip (cm) & $99.87 \pm 9.42$ & $103.55 \pm 8.78$ & $98.05 \pm 9.19$ & $<0.001$ \\
\hline Waist (cm) & $92.75 \pm 12.89$ & $102.15 \pm 9.23$ & $88.12 \pm 11.88$ & $<0.001$ \\
\hline Waist to hip & $0.92 \pm 0.08$ & $0.97 \pm 0.06$ & $0.88 \pm 0.07$ & $<0.001$ \\
\hline Triacylglycerol & $\begin{array}{l}136.45 \pm \\
119.68\end{array}$ & $196.80 \pm 182.31$ & $106.54 \pm 47.87$ & $<0.001$ \\
\hline Total cholesterol (mg/dl) & $185.94 \pm 44.02$ & $203.18 \pm 50.22$ & $177.40 \pm 37.84$ & $<0.001$ \\
\hline $\begin{array}{l}\text { High density lipoprotein cholesterol } \\
(\mathrm{mg} / \mathrm{dl})\end{array}$ & $48.20 \pm 11.22$ & $42.83 \pm 8.89$ & $50.84 \pm 11.31$ & $<0.001$ \\
\hline $\begin{array}{l}\text { Low density lipoprotein cholesterol } \\
(\mathrm{mg} / \mathrm{dl})\end{array}$ & $110.69 \pm 35.71$ & $121.72 \pm 37.55$ & $105.24 \pm 33.48$ & 0.126 \\
\hline Systolic blood pressure (mm Hg) & $115.21 \pm 18.75$ & $127.72 \pm 20.97$ & $109.05 \pm 13.92$ & $<0.001$ \\
\hline Diastolic blood pressure $(\mathrm{mm} \mathrm{Hg})$ & $74.86 \pm 10.59$ & $80.01 \pm 10.82$ & $72.31 \pm 9.51$ & $<0.001$ \\
\hline Glucose $(\mathrm{mg} / \mathrm{dL})$ & $100.64 \pm 28.40$ & $116.09 \pm 41.22$ & $92.98 \pm 13.76$ & $<0.001$ \\
\hline a) Mean $\pm S D$ & & & & \\
\hline
\end{tabular}


Table 2

Clinical, anthropometric, and laboratory measurements according to the gender of the participant

\begin{tabular}{|llll|}
\hline Parameters & Male $(\mathbf{n}=\mathbf{5 4 0 9})$ & Female $(\mathbf{n}=\mathbf{6 8 7 3})$ & P-value \\
\hline Age (year) & $43.01 \pm 20.00$ a) & $43.90 \pm 17.21$ & 0.07 \\
\hline Body mass index $\left(\mathrm{kg} / \mathrm{m}^{2}\right)$ & $26.67 \pm 4.70$ & $27.97 \pm 5.59$ & $<0.001$ \\
\hline Weight $(\mathrm{kg})$ & $76.56 \pm 15.21$ & $68.72 \pm 13.81$ & $<0.001$ \\
\hline Height $(\mathrm{cm})$ & $170.76 \pm 8.60$ & $155.46 \pm 6.48$ & $<0.001$ \\
\hline Hip $(\mathrm{cm})$ & $98.46 \pm 8.10$ & $101.13 \pm 9.78$ & 0.08 \\
\hline Waist $(\mathrm{cm})$ & $93.94 \pm 12.07$ & $92.35 \pm 13.18$ & 0.22 \\
\hline Waist to hip & $0.96 \pm 0.06$ & $0.89 \pm 0.09$ & $<0.001$ \\
\hline Triacylglycerol & $135.36 \pm 61.39$ & $136.57 \pm 148.88$ & 0.37 \\
\hline Total cholesterol (mg/dl) & $182.84 \pm 39.14$ & $188.81 \pm 47.75$ & 0.80 \\
\hline High density lipoprotein cholesterol (mg/dl) & $43.93 \pm 9.15$ & $51.30 \pm 11.47$ & $<0.001$ \\
\hline Low density lipoprotein cholesterol (mg/dl) & $111.83 \pm 34.52$ & $110.56 \pm 37.01$ & 0.49 \\
\hline Systolic blood pressure (mm Hg) & $116.86 \pm 16.10$ & $114.38 \pm 20.58$ & 0.11 \\
\hline Diastolic blood pressure (mm Hg) & $76.00 \pm 10.62$ & $74.27 \pm 10.44$ & 0.04 \\
\hline Glucose (mg/dL) & $101.75 \pm 25.48$ & $100.42 \pm 31.05$ & 0.26 \\
\hline a) Mean \pm SD & & & \\
\hline
\end{tabular}

Allele frequency of APOE- $\varepsilon 2$, APOE- $\varepsilon 3$, and APOE- $\varepsilon 4$ was $5.36 \%, 88.01 \%$, and $6.61 \%$ in subjects with MetS. The APOE3- $\varepsilon 3$ genotype showed the highest (79.6\%), and the APOE2- 22 genotype had the lowest frequency $(0.5 \%)$ in the controls. The APOE \&3.ع3 genotype was the most frequent in both the cases and the controls $(78.0 \%$ and $79.6 \%$, respectively) (Table 3$)$. 
Table 3

The $A P O E$ allele and genotype frequencies in case and control groups

\begin{tabular}{|c|c|c|c|}
\hline & Total $(n=9409)$ & With MetS ( $n=4449)$ & Without MetS $(n=4960)$ \\
\hline \multicolumn{4}{|c|}{ Genotype frequency (\%) } \\
\hline E2E2 & $46(0.4)$ & $22(0.5)$ & $24(0.5)$ \\
\hline E2E3 & $831(6.8)$ & 394 (8.9) & $437(8.8)$ \\
\hline E2E4 & $66(0.5)$ & $39(0.9)$ & $27(0.5)$ \\
\hline E3E3 & $7415(60.4)$ & $3468(78.0)$ & 3947 (79.6) \\
\hline E3E4 & $1006(8.2)$ & $502(11.3)$ & $504(10.2)$ \\
\hline E4E4 & $45(0.4)$ & $24(0.5)$ & $21(0.4)$ \\
\hline \multicolumn{4}{|c|}{ Allele frequency (\%) } \\
\hline$\varepsilon 2$ & $989(5.25)$ & $477(5.36)$ & $512(5.16)$ \\
\hline$\varepsilon 3$ & 16667(88.56) & 7832(88.01) & $8835(89.06)$ \\
\hline$\varepsilon 4$ & 1162(6.17) & $589(6.61)$ & $573(5.77)$ \\
\hline \multicolumn{4}{|l|}{ Carrier (\%) } \\
\hline$\varepsilon 2(\mathrm{E} 2 \mathrm{E} 2+\mathrm{E} 2 \mathrm{E} 3)$ & $877(45.50)$ & $416(44.16)$ & $461(46.75)$ \\
\hline$\varepsilon 4$ (E3E4 + E4E4) & $1051(54.50)$ & $526(55.84)$ & $525(44.25)$ \\
\hline
\end{tabular}

The association between the APOE genotypes and anthropometric measurements is shown in Table 4. The results indicated no significant difference between two different $A P O E$ genotype groups ( $\varepsilon 2$ vs. $\varepsilon 4$ carriers) and clinical, anthropometric, and laboratory parameters except low-density lipoprotein cholesterol (LDL). A different pattern was found between the cases and the controls at the genotype level. The results showed that the APOE genotypes significantly affect body mass index, weight, and hip in the male group (Table 5). However, no significant differences were observed between different $A P O E$ genotypes and anthropometric measurements. 
Table 4

Association between the $A P O E$ genotypes and anthropometric measurements in $\varepsilon 2$ and $\varepsilon 4$ carriers

\begin{tabular}{|c|c|c|c|c|c|c|c|c|c|}
\hline \multirow[t]{2}{*}{ Parameters } & \multicolumn{3}{|l|}{ Overall } & \multicolumn{3}{|c|}{ With MetS } & \multicolumn{3}{|c|}{ Without MetS } \\
\hline & $\begin{array}{l}E 2 \\
(\llbracket 2 \rrbracket 2 / \\
\bigotimes 2 \llbracket 3)\end{array}$ & $\begin{array}{l}E 4(\llbracket 3 \rrbracket 4 / \\
\bigotimes 4 囚 4)\end{array}$ & $\begin{array}{l}\text { P- } \\
\text { Value }\end{array}$ & $\begin{array}{l}E 2 \\
(₫ 2 \searrow 2 / \\
\bigotimes 2 \searrow 3)\end{array}$ & 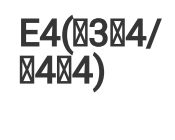 & $\begin{array}{l}\text { P- } \\
\text { Value }\end{array}$ & $\begin{array}{l}E 2 \\
(\llbracket 2 \rrbracket 2 / \\
\bigotimes 2 \bigotimes 3)\end{array}$ & 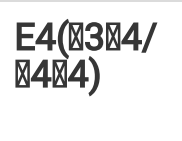 & $\begin{array}{l}\text { P- } \\
\text { Value }\end{array}$ \\
\hline & $\begin{array}{l}(n= \\
877)\end{array}$ & $\begin{array}{l}(n= \\
1051)\end{array}$ & & $\begin{array}{l}(n= \\
416)\end{array}$ & $(n=526)$ & & $\begin{array}{l}(n= \\
461)\end{array}$ & $(n=525)$ & \\
\hline Age (year) & $\begin{array}{l}45.62 \\
\pm \\
18.16^{a)}\end{array}$ & $\begin{array}{l}44.94 \pm \\
18.22\end{array}$ & 0.48 & $\begin{array}{l}55.34 \\
\pm 15.82\end{array}$ & $\begin{array}{l}54.28 \pm \\
15.25\end{array}$ & 0.35 & $\begin{array}{l}36.13 \\
\pm 15.02\end{array}$ & $\begin{array}{l}34.54 \pm \\
15.42\end{array}$ & 0.16 \\
\hline $\begin{array}{l}\text { Body mass } \\
\text { index }\left(\mathrm{kg} / \mathrm{m}^{2}\right)\end{array}$ & $\begin{array}{l}27.35 \\
\pm 5.01\end{array}$ & $\begin{array}{l}27.60 \pm \\
5.39\end{array}$ & 0.37 & $\begin{array}{l}29.77 \\
\pm 4.58\end{array}$ & $\begin{array}{l}30.13 \pm \\
5.01\end{array}$ & 0.13 & $\begin{array}{l}25.09 \\
\pm 4.34\end{array}$ & $\begin{array}{l}24.86 \pm \\
4.36\end{array}$ & 0.88 \\
\hline Weight (kg) & $\begin{array}{l}73.40 \\
\pm 14.73\end{array}$ & $\begin{array}{l}74.02 \pm \\
16.49\end{array}$ & 0.46 & $\begin{array}{l}79.55 \\
\pm 13.51\end{array}$ & $\begin{array}{l}80.62 \pm \\
15.34\end{array}$ & 0.33 & $\begin{array}{l}67.63 \\
\pm 13.46\end{array}$ & $\begin{array}{l}66.80 \pm \\
14.60\end{array}$ & 0.44 \\
\hline Height (cm) & $\begin{array}{l}163.75 \\
\pm 10.45\end{array}$ & $\begin{array}{l}163.57 \\
\pm 10.57\end{array}$ & 0.74 & $\begin{array}{l}163.55 \\
\pm 10.77\end{array}$ & $\begin{array}{l}163.63 \\
\pm 10.41\end{array}$ & 0.92 & $\begin{array}{l}163.93 \\
\pm 10.16\end{array}$ & $\begin{array}{l}163.50 \\
\pm 10.76\end{array}$ & 0.59 \\
\hline Hip (cm) & $\begin{array}{l}99.85 \\
\pm 10.20\end{array}$ & $\begin{array}{l}100.11 \\
\pm 10.52\end{array}$ & 0.87 & $\begin{array}{l}102.61 \\
\pm 9.62\end{array}$ & $\begin{array}{l}103.04 \\
\pm 10.49\end{array}$ & 0.47 & $\begin{array}{l}96.90 \\
\pm 9.54\end{array}$ & $\begin{array}{l}97.14 \pm \\
9.12\end{array}$ & 0.94 \\
\hline Waist (cm) & $\begin{array}{l}92.73 \\
\pm 12.58\end{array}$ & $\begin{array}{l}92.98 \pm \\
13.52\end{array}$ & 0.72 & $\begin{array}{l}100.12 \\
\pm 9.98\end{array}$ & $\begin{array}{l}100.37 \\
\pm 11.06\end{array}$ & 0.75 & $\begin{array}{l}85.79 \\
\pm 52.47\end{array}$ & $\begin{array}{l}114.74 \\
\pm 59.21\end{array}$ & 0.27 \\
\hline Waist to hip & $\begin{array}{l}0.93 \pm \\
0.08\end{array}$ & $\begin{array}{l}0.92 \pm \\
0.08\end{array}$ & 0.51 & & & & & & \\
\hline Triacylglycerol & $\begin{array}{l}156.24 \\
\pm 59.96\end{array}$ & $\begin{array}{l}147.68 \\
\pm 89.71\end{array}$ & 0.20 & $\begin{array}{l}200.01 \\
\pm \\
212.07\end{array}$ & $\begin{array}{l}177.59 \\
\pm 101.52\end{array}$ & 0.06 & $\begin{array}{l}113.02 \\
\pm 61.98\end{array}$ & $\begin{array}{l}101.82 \\
\pm 38.27\end{array}$ & 0.68 \\
\hline $\begin{array}{l}\text { High density } \\
\text { lipoprotein } \\
\text { cholesterol } \\
\text { (mg/dl) }\end{array}$ & $\begin{array}{l}47.44 \\
\pm 11.58\end{array}$ & $\begin{array}{l}45.81 \pm \\
11.02\end{array}$ & 0.007 & $\begin{array}{l}43.97 \\
\pm 10.64\end{array}$ & $\begin{array}{l}42.46 \pm \\
9.39\end{array}$ & 0.04 & $\begin{array}{l}50.85 \\
\pm 11.47\end{array}$ & $\begin{array}{l}49.49 \pm \\
11.51\end{array}$ & 0.11 \\
\hline $\begin{array}{l}\text { Low density } \\
\text { lipoprotein } \\
\text { cholesterol } \\
\text { (mg/dl) }\end{array}$ & $\begin{array}{l}96.62 \\
\pm 32.55\end{array}$ & $\begin{array}{l}113.78 \\
\pm 34.75\end{array}$ & 0.0001 & $\begin{array}{l}100.44 \\
\pm 32.13\end{array}$ & $\begin{array}{r}115.15 \\
\pm 36.62\end{array}$ & 0.0001 & $\begin{array}{l}92.97 \\
\pm 32.57\end{array}$ & $\begin{array}{l}112.32 \\
\pm 32.61\end{array}$ & 0.0001 \\
\hline $\begin{array}{l}\text { Systolic blood } \\
\text { pressure (mm } \\
\mathrm{Hg})\end{array}$ & $\begin{array}{l}114.03 \\
\pm 17.35\end{array}$ & $\begin{array}{l}113.92 \\
\pm 17.04\end{array}$ & 0.90 & $\begin{array}{l}122.54 \\
\pm 17.85\end{array}$ & $\begin{array}{l}121.27 \\
\pm 16.94\end{array}$ & 0.32 & $\begin{array}{l}105.82 \\
\pm 12.14\end{array}$ & $\begin{array}{l}105.84 \\
\pm 13.04\end{array}$ & 0.99 \\
\hline $\begin{array}{l}\text { Diastolic } \\
\text { blood } \\
\text { pressure (mm } \\
\mathrm{Hg})\end{array}$ & $\begin{array}{l}74.99 \\
\pm 10.18\end{array}$ & $\begin{array}{l}75.64 \pm \\
10.21\end{array}$ & 0.23 & $\begin{array}{l}78.81 \\
\pm 10.33\end{array}$ & $\begin{array}{l}78.71 \pm \\
9.77\end{array}$ & 0.89 & $\begin{array}{l}71.32 \\
\pm 8.57\end{array}$ & $\begin{array}{l}72.27 \pm \\
9.61\end{array}$ & 0.17 \\
\hline $\begin{array}{l}\text { Glucose } \\
\text { (mg/dL) }\end{array}$ & $\begin{array}{l}99.29 \\
\pm 29.04\end{array}$ & $\begin{array}{l}97.94 \pm \\
25.49\end{array}$ & 0.35 & $\begin{array}{l}109.58 \\
\pm 37.82\end{array}$ & $\begin{array}{l}107.01 \\
\pm 31.74\end{array}$ & 0.31 & $\begin{array}{l}89.13 \\
\pm 7.60\end{array}$ & $\begin{array}{l}87.98 \pm \\
8.29\end{array}$ & 0.05 \\
\hline
\end{tabular}


Table 5

Association between the APOE genotypes and anthropometric measurements in $\varepsilon 2 \varepsilon 2$ and $\varepsilon 4 \varepsilon 4$ genotypes

\begin{tabular}{|c|c|c|c|c|c|c|c|c|c|}
\hline \multirow[t]{2}{*}{ Parameters } & \multicolumn{3}{|l|}{ Overall } & \multicolumn{3}{|c|}{ With MetS } & \multicolumn{3}{|c|}{ Without MetS } \\
\hline & $\llbracket 2 \llbracket 2$ & $\llbracket 4 \llbracket 4$ & $\begin{array}{l}\text { P- } \\
\text { Value }\end{array}$ & 囚2凶2 & $\llbracket 4 \llbracket 4$ & $\begin{array}{l}\mathrm{P}- \\
\text { Value }\end{array}$ & $\llbracket 2 \llbracket 2$ & 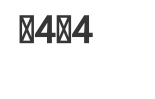 & $\begin{array}{l}\mathrm{P} \text { - } \\
\text { Value }\end{array}$ \\
\hline & $(n=46)$ & $(n=45)$ & & $\begin{array}{l}(n= \\
22)\end{array}$ & $(n=24)$ & & $\begin{array}{l}(n= \\
24)\end{array}$ & $\begin{array}{l}(n= \\
21)\end{array}$ & \\
\hline Age (year) & $\begin{array}{l}42.17 \pm \\
19.44^{a)}\end{array}$ & $\begin{array}{l}40.72 \\
\pm 18.74\end{array}$ & 0.76 & $\begin{array}{l}45.61 \\
\pm 19.44\end{array}$ & $\begin{array}{l}45.89 \pm \\
17.05\end{array}$ & 0.96 & $\begin{array}{l}38.53 \\
\pm 19.34\end{array}$ & $\begin{array}{l}32.27 \\
\pm 19.05\end{array}$ & 0.40 \\
\hline $\begin{array}{l}\text { Body mass index } \\
\left(\mathrm{kg} / \mathrm{m}^{2}\right)\end{array}$ & $\begin{array}{l}28.17 \pm \\
5.30\end{array}$ & $\begin{array}{l}28.22 \\
\pm 5.18\end{array}$ & 0.97 & $\begin{array}{l}31.27 \\
\pm 4.39\end{array}$ & $\begin{array}{l}30.65 \pm \\
5.54\end{array}$ & 0.71 & $\begin{array}{l}24.69 \\
\pm 3.92\end{array}$ & $\begin{array}{l}24.25 \\
\pm 5.17\end{array}$ & 0.80 \\
\hline Weight (kg) & $\begin{array}{l}77.21 \pm \\
15.67\end{array}$ & $\begin{array}{l}78.03 \\
\pm 21.23\end{array}$ & 0.85 & $\begin{array}{l}85.72 \\
\pm 13.55\end{array}$ & $\begin{array}{l}86.50 \pm \\
19.52\end{array}$ & 0.89 & $\begin{array}{l}67.63 \\
\pm 12.13\end{array}$ & $\begin{array}{l}64.18 \\
\pm 16.54\end{array}$ & 0.53 \\
\hline Height (cm) & $\begin{array}{l}165.20 \\
\pm 9.98\end{array}$ & $\begin{array}{l}165.48 \\
\pm 13.42\end{array}$ & 0.92 & $\begin{array}{l}165.56 \\
\pm 12.21\end{array}$ & $\begin{array}{l}167.94 \\
\pm 12.36\end{array}$ & 0.56 & $\begin{array}{l}164.82 \\
\pm 7.29\end{array}$ & $\begin{array}{l}161.45 \\
\pm 14.70\end{array}$ & 0.42 \\
\hline Hip (cm) & $\begin{array}{l}101.24 \\
\pm 8.36\end{array}$ & $\begin{array}{l}100.79 \\
\pm 11.34\end{array}$ & 0.86 & $\begin{array}{l}104.28 \\
\pm 7.89\end{array}$ & $\begin{array}{l}104.67 \\
\pm 9.47\end{array}$ & 0.89 & $\begin{array}{l}97.81 \\
\pm 7.73\end{array}$ & $\begin{array}{l}94.45 \\
\pm 11.68\end{array}$ & 0.37 \\
\hline Waist (cm) & $\begin{array}{l}94.56 \pm \\
13.12\end{array}$ & $\begin{array}{l}95.21 \\
\pm \\
14.576\end{array}$ & 0.63 & $\begin{array}{l}102.94 \\
\pm 9.40\end{array}$ & $\begin{array}{l}103.33 \\
\pm 10.75\end{array}$ & 0.90 & $\begin{array}{l}85.13 \\
\pm 9.96\end{array}$ & $\begin{array}{l}81.91 \\
\pm 9.97\end{array}$ & 0.41 \\
\hline Waist to hip & $\begin{array}{l}0.93 \pm \\
0.08\end{array}$ & $\begin{array}{l}0.94 \pm \\
0.07\end{array}$ & 0.63 & $\begin{array}{l}0.98 \pm \\
0.06\end{array}$ & $\begin{array}{l}0.98 \pm \\
0.04\end{array}$ & 0.97 & $\begin{array}{l}0.87 \pm \\
0.07\end{array}$ & $\begin{array}{l}0.86 \pm \\
0.05\end{array}$ & 0.96 \\
\hline Triacylglycerol & $\begin{array}{l}176.83 \\
\pm 80.40\end{array}$ & $\begin{array}{l}144.59 \\
\pm 92.70\end{array}$ & 0.14 & $\begin{array}{l}225.11 \\
\pm 72.39\end{array}$ & $\begin{array}{l}161.61 \\
\pm \\
107.78\end{array}$ & 0.04 & $\begin{array}{l}125.71 \\
\pm 52.61\end{array}$ & $\begin{array}{l}116.73 \\
\pm 54.22\end{array}$ & 0.66 \\
\hline $\begin{array}{l}\text { High density } \\
\text { lipoprotein } \\
\text { Cholesterol (mg/dl) }\end{array}$ & $\begin{array}{l}46.37 \pm \\
12.64\end{array}$ & $\begin{array}{l}43.20 \\
\pm 11.15\end{array}$ & 0.29 & $\begin{array}{l}41.11 \\
\pm 12.48\end{array}$ & $\begin{array}{l}41.11 \pm \\
10.33\end{array}$ & 0.98 & $\begin{array}{l}51.94 \\
\pm 10.53\end{array}$ & $\begin{array}{l}46.63 \\
\pm 12.07\end{array}$ & 0.23 \\
\hline $\begin{array}{l}\text { Low density } \\
\text { lipoprotein } \\
\text { cholesterol (mg/dl) }\end{array}$ & $\begin{array}{l}86.83 \pm \\
36.44\end{array}$ & $\begin{array}{l}109.69 \\
\pm 26.88\end{array}$ & 0.007 & $\begin{array}{l}91.86 \\
\pm 28.34\end{array}$ & $\begin{array}{l}110.71 \\
\pm \\
30.631\end{array}$ & 0.06 & $\begin{array}{l}81.50 \\
\pm 43.69\end{array}$ & $\begin{array}{l}108.10 \\
\pm 21.83\end{array}$ & 0.07 \\
\hline $\begin{array}{l}\text { Systolic blood } \\
\text { pressure }(\mathrm{mm} \mathrm{Hg})\end{array}$ & $\begin{array}{l}113.54 \\
\pm 13.37\end{array}$ & $\begin{array}{l}111.86 \\
\pm 15.48\end{array}$ & 0.64 & $\begin{array}{l}121.05 \\
\pm 12.73\end{array}$ & $\begin{array}{l}117.83 \\
\pm 14.43\end{array}$ & 0.48 & $\begin{array}{l}105.58 \\
\pm 8.80\end{array}$ & $\begin{array}{l}102.09 \\
\pm 12.12\end{array}$ & 0.38 \\
\hline $\begin{array}{l}\text { Diastolic blood } \\
\text { pressure }(\mathrm{mm} \mathrm{Hg})\end{array}$ & $\begin{array}{l}76.08 \pm \\
10.15\end{array}$ & $\begin{array}{l}78.13 \\
\pm 9.14\end{array}$ & 0.36 & $\begin{array}{l}81.11 \\
\pm 9.38\end{array}$ & $\begin{array}{l}82.22 \pm \\
6.99\end{array}$ & 0.69 & $\begin{array}{l}70.76 \\
\pm 8.19\end{array}$ & $\begin{array}{l}71.90 \\
\pm 8.84\end{array}$ & 0.72 \\
\hline Glucose (mg/dL) & $\begin{array}{l}92.29 \pm \\
10.77\end{array}$ & $\begin{array}{l}91.41 \\
\pm 9.13\end{array}$ & 0.87 & $\begin{array}{l}96.72 \\
\pm 11.41\end{array}$ & $\begin{array}{l}93.83 \pm \\
10.46\end{array}$ & 0.43 & $\begin{array}{l}87.59 \\
\pm 7.93\end{array}$ & $\begin{array}{l}87.45 \\
\pm 4.43\end{array}$ & 0.96 \\
\hline
\end{tabular}

The results indicated that the risk of Mets was not increased significantly in individuals with $\varepsilon 2 . \varepsilon 2$ genotypes compared to $\varepsilon 4 . \varepsilon 4$ genotypes $(\mathrm{OR}=1.24,95 \% \mathrm{Cl}=0.54-2.83)$. Stratifying the individuals into the male and female stratum showed that the $\varepsilon 4 . \varepsilon 4$ genotypes are associated with decreased risk of MetS $(\mathrm{OR}=0.80,95 \% \mathrm{Cl}=0.25-2.55)$. In contrast, a different magnitude was found in the female group ( $\mathrm{OR}=2.18,95 \% \mathrm{Cl}=0.64-7.40)$. In both cases, the results were not statistically different at $p$-value $<0.05$ (Table 6). 
Table 6

Association of the APOE genotypes with the risk of MetS

\begin{tabular}{|c|c|c|c|c|c|c|c|c|c|}
\hline \multirow[t]{2}{*}{ Genotype } & \multicolumn{3}{|c|}{ Overall } & \multicolumn{3}{|c|}{ Male } & \multicolumn{3}{|c|}{ Female } \\
\hline & OR & $95 \% \mathrm{Cl}$ & $\begin{array}{l}\text { P- } \\
\text { Value }\end{array}$ & OR & $95 \% \mathrm{Cl}$ & $\begin{array}{l}\text { P- } \\
\text { Value }\end{array}$ & OR & $95 \% \mathrm{Cl}$ & $\begin{array}{l}\text { P- } \\
\text { Value }\end{array}$ \\
\hline E2 /E4 carrier & 1.11 & $\begin{array}{l}0.92- \\
1.32\end{array}$ & 0.25 & 1.24 & $\begin{array}{l}0.95- \\
1.61\end{array}$ & 0.11 & 1.02 & $\begin{array}{l}0.79- \\
1.30\end{array}$ & 0.87 \\
\hline $\begin{array}{l}\text { E2E2/E4E4 } \\
\text { carrier }\end{array}$ & 1.24 & $\begin{array}{l}0.54- \\
2.83\end{array}$ & 0.59 & 0.80 & $\begin{array}{l}0.25- \\
2.55\end{array}$ & 0.71 & 2.18 & $\begin{array}{l}0.64- \\
7.40\end{array}$ & 0.21 \\
\hline
\end{tabular}

Search results and study inclusion

Potentially relevant studies selected through an initial literature search comprise 3228. After removing duplicate records within and between the databases, the total numbers of 2149 articles remained. Among these articles, 411 papers were excluded as they did not meet the selection criteria (supplementary table A.1). In the next step, the title and abstracts of 1783 remaining studies were screened based on the defined criteria, so 35 studies remained, and fulllength papers of these studies were retrieved and assessed for eligibility and methodological quality based on the designed form for full-text screening (supplementary table A.2). Studies that did not have a correct definition of the metabolic syndrome or reported irrelevant outcomes were excluded $(n=20)$. A total of 15 studies met inclusion criteria and were included in quantitate synthesis (Figure 1). The required data were recorded in a specific form and then prepared for data analysis using Stata software, version 14.

All included studies were published between 2009 and 2019 and had a sample size ranging from 147 to 1717 (Table $7)$. Of the 15 studies $(n=4425)$ included in this systematic review, five studies reported all types of APOE genotypes, and one did not report genotypes completely. Two studies investigated the ATP definition $(n=896)$, and one study applied the IDF definition $(n=383)$. Also, three studies investigated more than one definition of metabolic syndrome in their study $(n=3164)$.

Based on the results, among these 15 studies, one reported a significant, positive association between the carriers of the APOE- $\varepsilon 4$ allele and the risk of Mets. There was also only one study that reported the non-significant association between the APOE- $\varepsilon 4$ allele and the risk of MetS. With evidence of considerable heterogeneity $\left(I^{2}=30.2 \%\right)$, the APOE$\varepsilon 4$ allele could not increase the risk in MetS carriers in comparison with non-carriers (overall OR=0.97, 95\% Cl:0.841.09) (Figure 2). There was no evidence of publication bias according to the visual evaluation of the funnel plot (Figure 3). The Begg's test result approved the absence of publication bias $(P=0.88)$. The same result was obtained when Egger's test was used $(P=0.74)$. The results of $A P O E-\varepsilon 4 \varepsilon 4$ genotype and risk of Mets showed the same results as the APOE- $\varepsilon 4$ allele. The risk of metabolic syndrome increased in individuals with APOE- $\varepsilon 4 \varepsilon 4$ genotype compared to individuals with APOE- $\varepsilon 2 \varepsilon 2$ genotype. However, this association was not significant (overall OR=1.46, 95\% Cl: 0.58$3.64)$ with evidence of moderate heterogeneity $\left(I^{2}=54.8 \%\right)$ (Figure 4$)$. The results of Begg's test provided no evidence of publication bias $(P=0.48)$, which was in line with both Egger's test result $(P=0.40)$ and the funnel plot (Figure 5). The sensitivity analysis result indicated that the overall OR differed across different analyses (Figure 6 and 7). It implies that separate exclusion and inclusion criteria for eligible studies can lead to different findings. Therefore, the result may need to be interpreted with caution.

Table 7: Summary of eligible studies for meta-analysis

Page $10 / 16$ 


\begin{tabular}{llllll} 
Author & Year of publication & Country & Definition of MetS & Cases(n) & Controls(n) \\
\hline Daneshpour & NA $^{*}$ & Iran & JIS & 5696 & 6586 \\
\hline Feitosa & 2011 & USA & NCEP & 301 & 191 \\
\hline Ferreira & 2011 & Brazil & AHA & 1073 & 1244 \\
\hline Hughes & 2017 & USA & NCEP & 116 & 31 \\
\hline Jeenduang & 2015 & Thailand & NCEP ATPIII & 46 & 233 \\
\hline Karmelić & 2016 & Croatia & IDF & 122 & 122 \\
\hline Lai & 2016 & USA & AHA & 177 & 91 \\
\hline Lee. D & 2011 & Korean & NCEP /ATP III & 266 & 177 \\
\hline Lian Lai & 2014 & Taiwan & NAEPATPIII & 719 & 301 \\
\hline Novotny & 2014 & Czech Republic & NCEP ATPIII & 146 & 444 \\
\hline Ranjith & 2009 & Africa & NCEP III/ATP III & 65 & 144 \\
\hline Tao & 2011 & China & NCEP/IDF & 121 & 257 \\
\hline Teixeira & 2014 & Brazilian & IDF & 34 & 115 \\
\hline Torres-Perez & 2016 & Spain & IDF & 482 & 510
\end{tabular}

${ }^{*}$ Not applicable

\section{Discussion}

The current study consists of many case-control studies published since 1985 and pools the genetic data collected from 16,707 people residing in 16 countries. The results indicated no significant increased risk of metabolic syndrome in carriers of the $A P O E \& 4$ allele. According to the findings, the pooled estimate for MetS risk in the $\varepsilon 4$ carriers was 0.97 (95\% Cl: $0.84-1.09)$. This risk increased up to $46 \%$ in the presence of two copies of this allele $(\varepsilon 4 / 4)$. However, it was not statistically significant $(\mathrm{OR}=1.46,95 \% \mathrm{Cl}: 0.58-3.64)$.

In the TCGS population, the prevalence of MetS in individuals with the $\varepsilon 4 / 4$ genotype was higher than individuals carrying two copies of the $A P O E \varepsilon 2$ allele. Despite the relatively large sample size, the findings were not significant. The results also showed that the $A P O E \varepsilon 3 / 3$ was the predominant genotype among all studies with a frequency of $71.8 \%$, while $\varepsilon 2 / 2$ was less common. The same pattern was observed in the TCGS population, where the frequency of the $\varepsilon 3 / 3$ genotype in MetS and healthy groups was $78.0 \%$ and $79.6 \%$, respectively. The results implied that the frequency of $A P O E \& 3 / 3$ genotype in the TCGS population is between the distributions in American and European populations.

Besides the lowest regional estimates for $\varepsilon 4$ carrier status in Asia (41.9\%) and Southern Europe (40.5\%), most MetS cases were not $A P O E \& 4$ carriers. In marked contrast, the majority of the cases in Northern Europe were carriers (61.3\%). Estimating for APOE \&4/4 genotype was also observed in similar patterns. These trends are consistent with general population studies in which the $A P O E \varepsilon 4$ frequencies observe to be higher in Northern than in Southern Europe or Asia and to be lowest in Italy, Japan, and Korea[6-8]. 
Small-area variations in allele frequency, environmental risks that vary between regions, and methodologies may result in differences. At present, different criteria may use for the diagnosis of MetS in which the WHO, NCEP-ATP III, and IDF are the most widely used ones $[12,13]$. Since different criteria might generate different results, the prevalence of MetS varies depending on different diagnostic criteria. According to the previous reports, both IDF and NCEP-ATP III often use for diagnosis of MetS; however, NCEP-ATP III is the most frequently used criterion. Moreover, the prevalence of MetS is frequently higher based on IDF compared to NCEP-ATP III [14]. Therefore, this difference can consider as a potential source of bias in the data analysis process.

In our study, a total of 10 studies applied the NCEP-ATP III diagnostic criterion. This result reveals that NCEP-ATP III might be more appropriate for MetS diagnosis than IDF or WHO criteria. For further investigation of potential heterogeneities that exist among different studies, a subgroup analysis performed using stratification of a diagnostic criterion for MetS. Unexpectedly, no significant difference was found on the risk of MetS between the patients carrying the risk allele and healthy controls $(P>0.05)$. This result can be explained by an unequal distribution of articles within geographic locations. Considering only two studies with WHO criteria, it is impossible to conclude whether the diagnostic criterion is a confounder factor for substantial heterogeneity but suggests more studies are required in relevant countries.

Concerning sex composition, the current meta-analysis did not consider this factor for subgroup analysis; however, this variable might influence the overall result [15]. Due to either the limited or inadequate data, the sex subgroup could not be extracted from the final studies. Therefore, we failed to estimate whether males were more likely to develop MetS in comparison with females. The next point is that race was not recorded in many studies, and the ability to fully explore age was limited to the mean age reported in the publications.

Even though 15 eligible studies were all with suitable sample sizes and almost high quality, this meta-analysis has several limitations. First, several confounding factors may affect the association between the APOE and the risk of MetS. However, several confounding factors were adjusted in some studies; the results could still be affected by certain unadjusted confounding factors. Second, significant heterogeneity existed across studies, which might cause some bias in the final result. Since the reasons for the substantial heterogeneity were not possible to elucidate from this review, caution is warranted when attempting to infer regional or country-specific estimates of the association between the APOE $\varepsilon 4$ allele frequency and risk of MetS.

In conclusion, a high risk of MetS is not significantly associated with APOE alleles, especially when using the NCEPATP III diagnostic criterion. Although APOE \&4 is a well-studied genetic risk factor for developing MetS, most patients do not carry this genotype in some populations. Therefore, additional research is needed to understand both other genetic and environmental risk factors for MetS. The next point is that the genetic testing of $A P O E \varepsilon 4$ carrier status is not routinely considered in clinical practice. However, if the mechanism of action defines clearly, then there may be regions where testing could be essential for the early detection of individuals with a high risk of MetS.

\section{Declarations}

\section{Ethics approval and consent to participate}

The ethics committee of the Research Institute for Endocrine Sciences approved the study. All contributors signed informed written consent

\section{Availability of fata and materials}


The data analyzed during the current study for the Iranian population are available from the corresponding author on reasonable request.

\section{Competing interests}

The authors declare that they have no conflicts of interest.

\section{Authors' contributions}

MS, B.S.KH, and MSF conceived and designed the study. MS, AE, and LNHB analyzed the data and wrote the manuscript. MSD and FA supervised the project and edited the manuscript. All authors reviewed and approved the final version of the manuscript.

\section{Consent for Publication}

All authors give their consent for the publication

\section{Funding}

No funding was received

\section{Acknowledgments}

The authors would like to express their gratitude to the participants in the TCGS project. Also, special thanks for the scientific and financial support of the deCODE genetic company (Reykjavik, Ice-land).

\section{References}

1. Mahley RW, Apolipoprotein E. cholesterol transport protein with expanding role in cell biology. Science. 1988;240(4852):622-30.

2. Rall S, Weisgraber KH, Mahley RW. Human apolipoprotein E. The complete amino acid sequence. J Biol Chem. 1982;257(8):4171-8.

3. Mahley RW, Rall SC Jr, Apolipoprotein E. far more than a lipid transport protein. Annu Rev Genom Hum Genet. 2000;1(1):507-37.

4. Liberopoulos EN, et al. The influence of serum apolipoprotein E concentration and polymorphism on serum lipid parameters in hemodialysis patients. American journal of kidney diseases. 2004;44(2):300-8.

5. Lee D-J, et al. ApoE polymorphism may determine low-density lipoprotein cholesterol level in association with obesity and metabolic syndrome in postmenopausal Korean women. Yonsei Med J. 2011;52(3):429-34.

6. Seshasai RK, et al. Apolipoprotein E and kidney function in older adults. Clin Nephrol. 2012;78(3):174.

7. Leite MLC, Lima-Costa MF, Moriguchi EH. Age-related trends of blood pressure levels by apolipoprotein $\mathrm{E}$ genotype: the Bambuì cohort study of ageing (1997-2008). Hypertens Res. 2013;36(3):270-6.

8. Yin Y-W, et al. Influence of apolipoprotein E gene polymorphism on development of type 2 diabetes mellitus in Chinese Han population: a meta-analysis of 29 studies. Metabolism. 2014;63(4):532-41.

9. Crunkhorn S. Neurodegenerative disorders: Linking apolipoprotein E to LOAD. Nat Rev Drug Discovery. 2013;12(9):664-5.

10. Song Y, Stampfer MJ, Liu S. Meta-analysis: apolipoprotein E genotypes and risk for coronary heart disease. Ann Intern Med. 2004;141(2):137-47. 
11. Ferreira $D$, et al. association of apoliprotein E polymorphisms and metabolic syndrome in subjects with extreme obesity. Clin Chim Acta. 2011;412(17):1559-62.

12. Daneshpour MS, et al., Rationale and design of a genetic study on cardiometabolic risk factors: protocol for the Tehran Cardiometabolic Genetic Study (TCGS). JMIR research protocols, 2017. 6(2): p. e28.

13. Azizi F, et al. Prevention of non-communicable disease in a population in nutrition transition: Tehran Lipid and Glucose Study phase II. Trials. 2009;10(1):5.

14. Alberti $\mathrm{K}$, et al. Harmonizing the metabolic syndrome: a joint interim statement of the international diabetes federation task force on epidemiology and prevention; national heart, lung, and blood institute; American heart association; world heart federation; international atherosclerosis society; and international association for the study of obesity. Circulation. 2009;120(16):1640-5.

15. Hosseini-Esfahani F, et al., Metabolic syndrome: Findings from 20 years of the Tehran Lipid and Glucose Study. International journal of endocrinology and metabolism, 2018. 16(4 Suppl).

16. Miller S, Dykes D. and H.J.N.a.r. Polesky, A simple salting out procedure for extracting DNA from human nucleated cells. 1988. 16(3): p. 1215.

17. Daneshpour MS, et al. Rationale and Design of a Genetic Study on Cardiometabolic Risk Factors: Protocol for the Tehran Cardiometabolic Genetic Study (TCGS). JMIR Res Protoc. 2017;6(2):e28.

18. Azizi F, et al. Prevention of non-communicable disease in a population in nutrition transition: Tehran Lipid and Glucose Study phase II. Trials. 2009;10:5.

19. Alberti KG, et al., Harmonizing the metabolic syndrome: a joint interim statement of the International Diabetes Federation Task Force on Epidemiology and Prevention; National Heart, Lung, and Blood Institute; American Heart Association; World Heart Federation; International Atherosclerosis Society; and International Association for the Study of Obesity. Circulation, 2009. 120(16): p. 1640-5.

20. Furlan AD, et al., 2009 updated method guidelines for systematic reviews in the Cochrane Back Review Group. 2009. 34(18): p. 1929-1941.

\section{Supplementary}

Supplementary Tables are not available with this version.

\section{Figures}




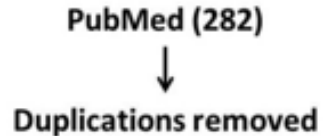

(141)
Scopus (646)

Duplications removed

(215)
Google Scholar (2300)

Duplications removed

(2088)

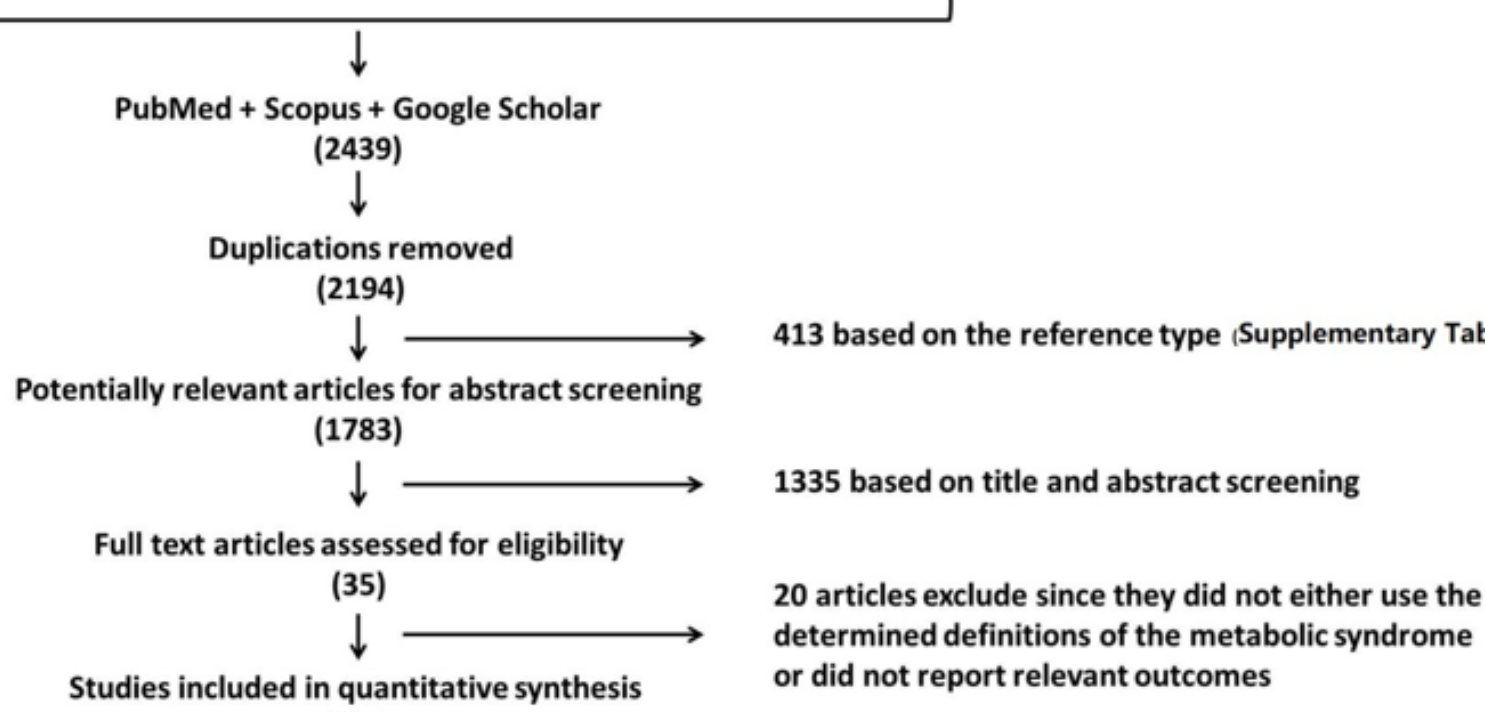

(15)

\section{Figure 1}

Flow diagram showing study selection process for meta-analysis

A)

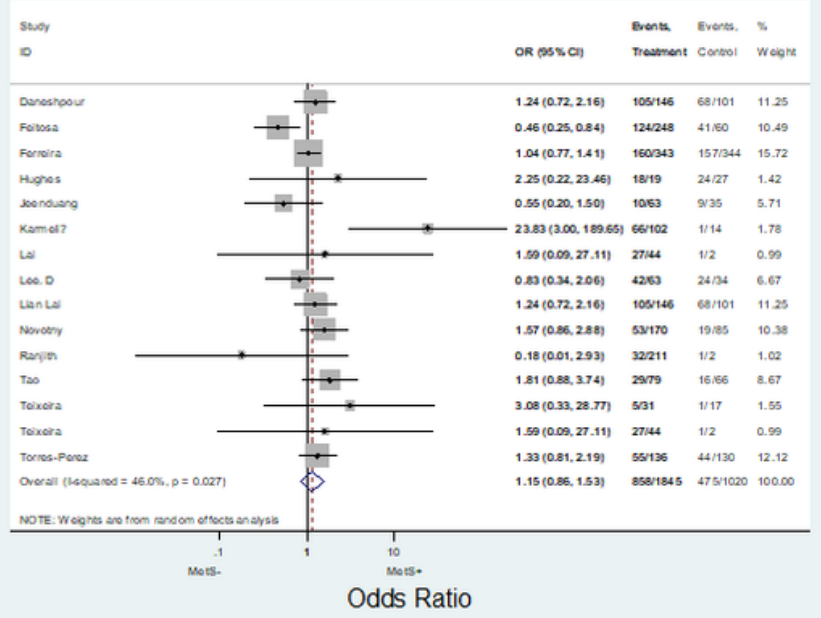

B)

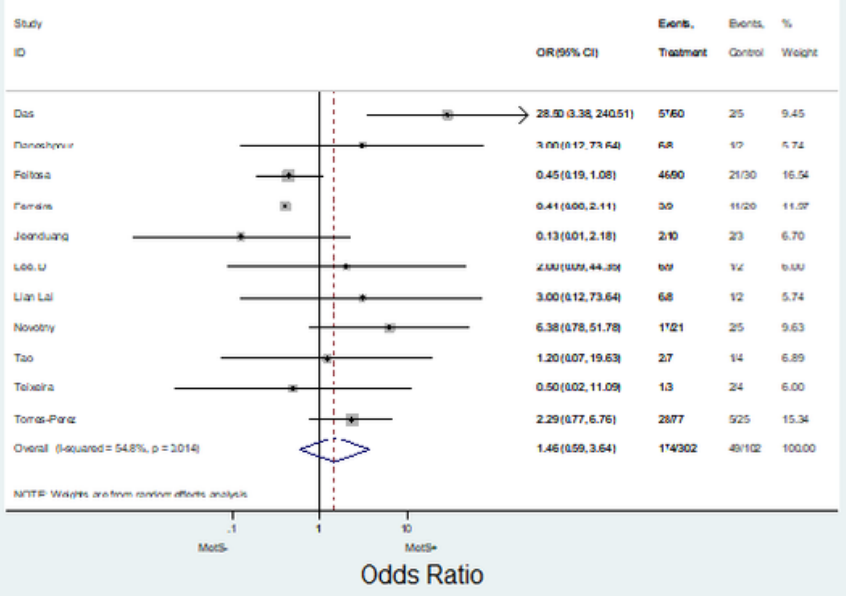

Figure 2

Forest plot for studies on A) APOE- $\varepsilon 4$ carriers and risk of MetS, B) APOE- $\varepsilon 4 \varepsilon 4$ genotype and risk of MetS 
A)

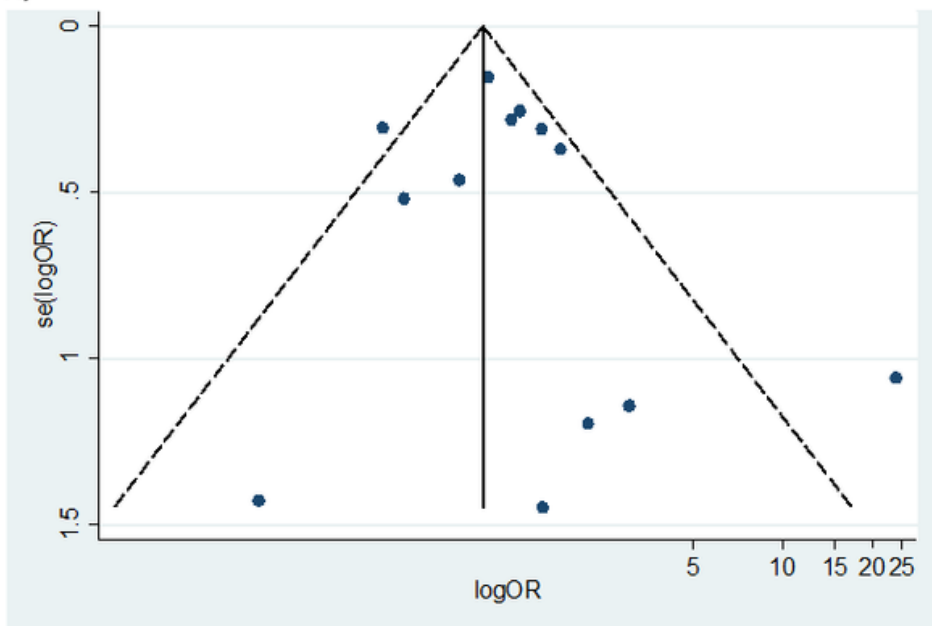

B)

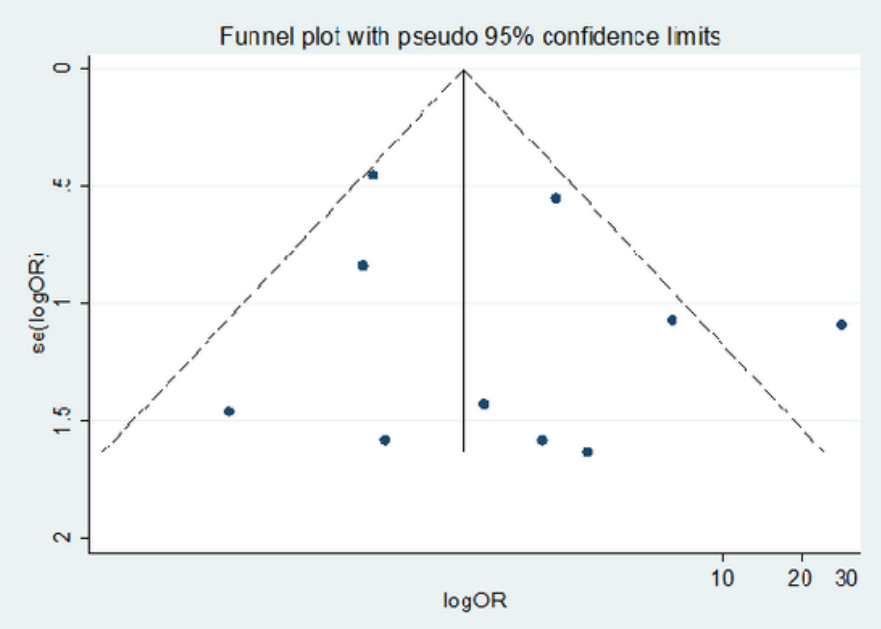

\section{Figure 3}

Funnel plot with pseudo 95\% confidence limits for studies on A) APOE- $\varepsilon 4 \varepsilon 4$ genotype and risk of MetS , B) APOE- $\varepsilon 4$ carriers and risk of MetS

A)

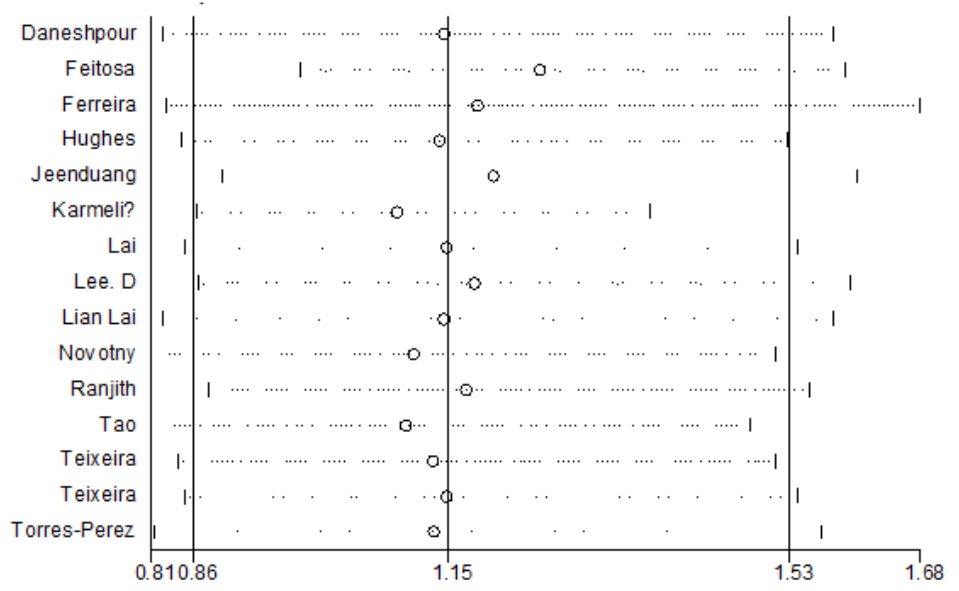

B)

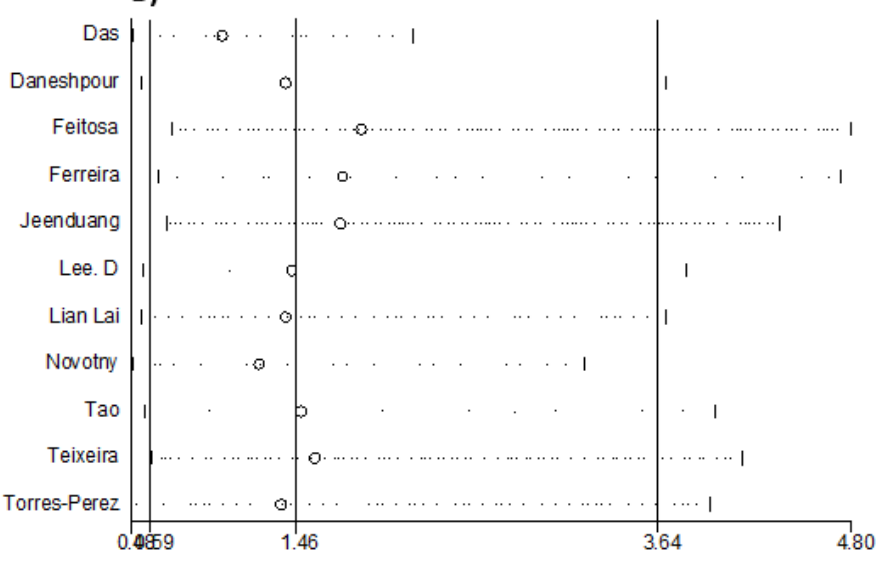

Figure 4

Sensitivity analysis for studies on A) APOE- $\varepsilon 4 \varepsilon 4$ genotype and risk of MetS, B) APOE- $\varepsilon 4$ carriers and risk of MetS 